\section{Low-complexity search method for CFO estimation in GFDM}

\author{
H. Shayanfar, H. Saeedi-Sourck ${ }^{\bowtie}$ and A. Farhang
}

In this Letter, the authors propose a low-complexity search method for carrier frequency offset (CFO) estimation in generalised frequency division multiplexing (GFDM). The proposed technique does not have any limitations on CFO acquisition range while providing an accurate estimate. Compared with the existing solutions in the literature with the lowest complexity, the proposed technique brings at least an order of magnitude complexity reduction without any performance penalty. Finally, the numerical results and comparisons with the existing literature in terms of performance and complexity attest the efficacy of the proposed method.

Introduction: One of the main challenges in multicarrier systems such as generalised frequency division multiplexing (GFDM) is their sensitivity to carrier frequency offset (CFO), caused by Doppler effect and local oscillator (LO) imperfections [1]. In typical wireless systems, LO accuracy is usually in the order of parts-per-million of the carrier frequency. Hence, the amount of CFO that is imposed by the LO misalignments in the emerging mmWave systems can be increased by orders of magnitude compared with sub- $6 \mathrm{GHz}$ systems. There have been a few recent attempts in the literature that address the CFO estimation in GFDM. In [1], the well-known Schmidl and Cox synchronisation technique [2] is adopted to GFDM. In [3], a blind maximum likelihood (ML) $\mathrm{CFO}$ and timing offset (TO) synchronisation technique have been proposed. Na et al. [4] designed a preamble consisting of two identical pseudo-noise (PN) sequences for TO and CFO estimation. However, all the estimation methods in [1-4] are limited to the CFO acquisition range of only half subcarrier spacing. To address this issue, a few solutions in the literature have been proposed [5-7]. Li et al. [5] proposed a joint CFO and channel estimation method which can be straightforwardly extended to GFDM. However, its performance highly depends on the type of the training sequence being deployed and it is very complex for implementation in practical systems. More recently, the authors in $[6,7]$ proposed joint ML-based CFO and channel estimation techniques with concentrating on the complexity issue which take the particular advantages of the Zaduff-Chu (ZC) sequences.

In this Letter, we deploy a preamble block for GFDM containing two similar frequency-domain ZC (FD-ZC) sequences at the beginning of each data packet. Deployment of this particular training sequence leads to a cost function for the ML-based CFO estimator in [6] that is sparse. Consequently, the search space will be reduced to a small number of search regions. Therefore, we propose a search method that leads to around an order of magnitude complexity reduction without any performance penalty compared with the solutions in [5-7]. We demonstrate superiority of our proposed method over the solutions in [5-7] through computational complexity analysis and performance comparisons using simulations.

System model: Let $M$ and $N$ be the total number of GFDM symbols and subcarriers in each GFDM block, respectively. According to the frequency spreading GFDM transmitter structure in [6], the GFDM transmit signal can be constructed as

$$
\boldsymbol{x}=\mathcal{F}_{M N}^{\mathrm{H}}\left(\operatorname{diag}\left(\boldsymbol{a}_{0}\right) \boldsymbol{C} \boldsymbol{d}_{\mathrm{e}}[0]+\cdots+\operatorname{diag}\left(\boldsymbol{a}_{M-1}\right) \boldsymbol{C} \boldsymbol{d}_{\mathrm{e}}[M-1]\right),
$$

where $\operatorname{diag}\left(\boldsymbol{a}_{m}\right)$ is a diagonal matrix whose diagonal elements include the elements of the vector $\boldsymbol{a}_{m}=\left[1, e^{-(j 2 \pi m / M)}, \ldots\right.$, $\left.e^{-(j 2 \pi m / M)(M N-1)}\right]^{\mathrm{T}}, \boldsymbol{C}$ is an $M N \times M N$ circulant matrix with the first column $\boldsymbol{c}=\left[\begin{array}{lllll}c_{0} & c_{1} \cdots c_{M-1} & 0 \cdots 0 & c_{M-1} \cdots c_{1}\end{array}\right]^{\mathrm{T}}$ containing the $2 M-1$ non-zero frequency domain coefficients of the prototype filter, and the $M N \times 1$ vector $\boldsymbol{d}_{\mathrm{e}}[m]$ is the $M$-fold expanded version of the column vector $d[m]=\left[d_{0, m}, \ldots, d_{N-1, m}\right]^{\mathrm{T}}$, with the entries $d_{n, m}$ corresponding to the data symbol to be transmitted on the $n$th subcarrier and the $m$ th time slot. Finally, $\mathcal{F}_{M N}$ is the $M N$-point normalised discrete Fourier transform matrix with the elements $\left[\mathcal{F}_{M N}\right]_{k \ell}=$ $(1 / \sqrt{M N}) e^{-j(2 \pi k \ell / M N)}$. In GFDM, a cyclic prefix $(\mathrm{CP})$ which is longer than the channel delay spread is appended to the beginning of the block, $\boldsymbol{x}$, prior to transmission to accommodate the channel transient period. This enables frequency-domain equalisation to tackle the wireless channel impairments and reduce the channel equalisation complexity.
The $M N \times 1$ received signal vector $\boldsymbol{r}$ that has gone through the channel, after $\mathrm{CP}$ removal can be written as $\boldsymbol{r}=\boldsymbol{E X} \boldsymbol{h}+\boldsymbol{v}$ where $\boldsymbol{v} \sim \mathcal{C N}\left(0, \sigma_{v}^{2} \boldsymbol{I}_{M N}\right)$ is the complex additive white Gaussian noise vector with the variance $\sigma_{v}^{2}$. The vector $\boldsymbol{h}=\left[h_{0}, \ldots, h_{L-1}\right]^{\mathrm{T}}$ is the channel impulse response (CIR) with the length $L$, and $\boldsymbol{E}=\operatorname{diag}(\boldsymbol{\varphi})$ is the $M N \times M N$ CFO matrix where $\varphi=\left[1, e^{j 2 \pi \varepsilon / N}, \ldots\right.$, $\left.e^{(j 2 \pi \varepsilon(M N-1)) / N}\right]^{\mathrm{T}}$ and $\varepsilon$ is the normalised CFO to the subcarrier spacing. Additionally, the $M N \times L$ matrix $\boldsymbol{X}$ contains the first $L$ columns of a circulant matrix whose first column equals to the GFDM transmit signal $\boldsymbol{x}$.

Proposed CFO estimation: Based on the results of previous section, CIR coefficients can be calculated as $\hat{\boldsymbol{h}}=\left(\boldsymbol{X}^{\mathrm{H}} \boldsymbol{X}\right)^{-1} \boldsymbol{X}^{\mathrm{H}} \boldsymbol{E}^{\mathrm{H}} \boldsymbol{r}$. Substituting $\boldsymbol{h}$ with $\hat{\boldsymbol{h}}$ in $\boldsymbol{r}=\boldsymbol{E} \boldsymbol{X} \boldsymbol{h}+\boldsymbol{v}$, the ML-based CFO estimate can be obtained as

$$
\hat{\varepsilon}=\underset{\tilde{\varepsilon}}{\operatorname{argmax}}\left\{\boldsymbol{r}^{\mathrm{H}} \widetilde{\boldsymbol{E}} \boldsymbol{X}\left(\boldsymbol{X}^{\mathrm{H}} \boldsymbol{X}\right)^{-1} \boldsymbol{X}^{\mathrm{H}} \widetilde{\boldsymbol{E}}^{\mathrm{H}} \boldsymbol{r}\right\},
$$

where $\widetilde{\boldsymbol{E}}$ is obtained in the same way as $\boldsymbol{E}$ by substitution of $\tilde{\varepsilon}$ rather than $\varepsilon$. However, using (2), an accurate CFO estimate can only be achieved through exhaustive search with a very small step-size in the range $|\tilde{\varepsilon}| \leq N / 2$. This can lead to a very large search space and thus a high computational load. To solve this issue and lower the complexity, in this Letter, we feed two similar FD-ZC sequences into the GFDM modulator to form the preamble block. Additionally, using $M=2$ for the preamble reduces the training overhead. It is worth to note that a $\mathrm{ZC}$ sequence with the length $N$ is defined as $z=1 / \sqrt{N}\left[1, e^{j \beta \pi / N}\right.$ $\left.\ldots, e^{(j \beta \pi / N)(N-1)^{2}}\right]^{\mathrm{T}}$, where $\beta$ is an integer parameter relatively prime with respect to $N$. Thus, the FD-ZC sequence can be obtained as $\boldsymbol{\psi}=\mathcal{F}_{N} \boldsymbol{z}$. According to (1), it can be shown that the orthogonal property of the FD-ZC sequence is preserved for the transmitted signal and the matrix $\boldsymbol{X}^{\mathrm{H}} \boldsymbol{X}$ becomes diagonal [7]. Consequently, equation (2) reduces to

$$
\hat{\varepsilon}=\underset{\tilde{\varepsilon}}{\operatorname{argmax}}\left\{\left\|\boldsymbol{r}^{\mathrm{H}} \widetilde{\boldsymbol{E}} \boldsymbol{X}\right\|_{2}\right\} .
$$

where $\hat{\varepsilon}$ can be obtained by maximising the cost function $\mathcal{G}=\left\|\boldsymbol{r}^{\mathrm{H}} \widetilde{\boldsymbol{E}} \boldsymbol{X}\right\|_{2}$ For the reasons that will be explained shortly, the main benefit of our proposed training sequence is that it makes the cost function $\mathcal{G}$ sparse. This dramatically reduces the search space size and leads to a substantial complexity reduction.

At high SNRs, $\boldsymbol{r} \simeq \boldsymbol{E} \boldsymbol{X} \boldsymbol{h}$ and (3) is equal to $\hat{\varepsilon}=$ $\operatorname{argmax}_{\tilde{\varepsilon}}\left\{\left\|\boldsymbol{h}^{\mathrm{H}} \boldsymbol{X}^{\mathrm{H}} \boldsymbol{\Phi} \boldsymbol{X}\right\|_{2}\right\}, \quad$ where $\quad \boldsymbol{\Phi}=\widetilde{\boldsymbol{E}}^{\mathrm{H}} \boldsymbol{E}=\operatorname{diag}\left(\left[\begin{array}{ll}\boldsymbol{\xi}^{\mathrm{T}} & \lambda \boldsymbol{\xi}^{\mathrm{T}}\end{array}\right]^{\mathrm{T}}\right)$ with $\boldsymbol{\xi}=\left[1, e^{j 2 \pi \Delta f / N}, \ldots, e^{(j 2 \pi \Delta f(N-1)) / N}\right]^{\mathrm{T}}, \quad \Delta f=\tilde{\varepsilon}-\varepsilon \quad$ and $\lambda=e^{j 2 \pi \Delta f}$. Concentrating on the term $\boldsymbol{Q} \triangleq \boldsymbol{X}^{\mathrm{H}} \boldsymbol{\Phi} \boldsymbol{X}$, it can be shown that the elements $[Q]_{l_{1}, l_{2}}$ in the $L \times L$ matrix are obtained as

$$
[\boldsymbol{Q}]_{l_{1}, l_{2}}=\rho e^{\left(j \beta \pi\left(l_{2}{ }^{2}-l_{1}{ }^{2}\right)\right) / N} \sum_{n=0}^{N-1} e^{\left(-j 2 \pi\left(\beta l_{1}-\beta l_{2}+\Delta f\right) n\right) / N},
$$

where $\rho=2 c_{0}^{2}(1+\lambda), \Delta f=\Delta f_{\mathrm{i}}+\Delta f_{\mathrm{f}}$, and $\Delta f_{\mathrm{i}}$ and $\Delta f_{\mathrm{f}}$ are the integer and fractional parts of $\Delta f$, respectively. Assuming $\Delta f_{\mathrm{f}}=0$ and $-N / 2 \leq \Delta f_{\mathrm{i}} \leq N / 2-1$, we can conclude that (4) is non-zero only when $\Delta f_{\mathrm{i}}=\beta\left(l_{2}-l_{1}\right)$, see Fig. 1 (note that this figure is obtained when the search step-size is $\gamma=1$ ). Hence, the cost function $\mathcal{G}$ has $2 L-1$ local peaks that are spaced $\beta$ positions away from each other. This feature of the cost function is the key to our proposed algorithm that achieves an accurate CFO estimation with a very low computational cost. Our proposed search method, summarised in Algorithm 1, involves two stages, namely, coarse estimation and fine estimation. At the coarse estimation stage, we set the search step-size to a value larger than one to reduce the computational cost. This is due to the fact that we only need to find the local peaks. According to Fig. 1, by setting the search stepsize in the range $L<\gamma \leq 2 L-1$, we can find at least one local peak. However, a very small peak out of the range (A $-\beta \times L / 2$, $\mathrm{A}+\beta \times L / 2)$ that may be buried in the noise may be found which will lead to an inaccurate CFO estimate. Hence, the largest search stepsize that can be used to achieve a reliable CFO estimate is $\gamma=L$. Using this step-size, we find the location of one of the local peaks that maximises $\mathcal{G}$. Knowing that the spacing of $\beta$ between the peaks, we will find $\lceil\gamma / 2\rceil$ peaks at each side of the peak that was found in step 1 of Algorithm 1, where $\lceil\cdot\rceil$ is the round-up operator. Then, we will locally search the neighbourhood of one subcarrier spacing around each of these peaks with the step-size of $10 \%$ the subcarrier spacing and find the coarse $\mathrm{CFO}$ estimate, $\hat{\varepsilon}_{0}$ that maximises $\mathcal{G}$. Finally, at the 
fine estimation stage, we search the neighbourhood of $\hat{\varepsilon}_{0}$ in $q$ iterations to achieve a CFO estimate with an accuracy of $\delta=10^{-(q+1)}$.

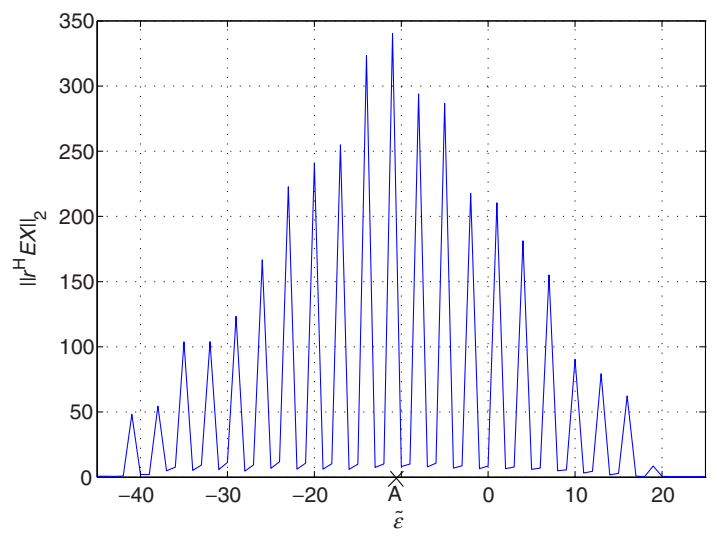

Fig. 1 Cost function $\mathcal{G}$ with $N=128, L=11, \varepsilon=-11.1283$ and $\beta=3$

Algorithm 1 (Proposed search method for CFO estimation):

\section{Initialisation:}

Set the primary search step: $\gamma=L^{*}$

Set the search tolerance: $\delta=10^{-(q+1)}$

Algorithm:

Step 2: Determine $\psi_{i}=\psi_{0}+\beta i$, for $i= \pm\{1,2, \ldots,\lceil\gamma / 2\rceil\}$, i.e. the peaks located at the neighbourhood of $\psi_{0}$.

Step 3: $\hat{\varepsilon}_{0}=\underset{\sim}{\operatorname{argmax}}\{\mathcal{G}\} \quad$ where $\tilde{\psi} \in \bigcup_{i}\left[\psi_{i}-0.5, \psi_{i}+0.5\right)$, for $\psi$

$i= \pm\{0,1, \ldots,\lceil\gamma / 2\rceil\}$ with the search step 0.1. (Coarse estimation)

Step 4: for $n=1$ to $q, \hat{\varepsilon}_{n}=\underset{\tilde{\varepsilon}}{\operatorname{argmax}}\{\mathcal{G}\}, \quad$ where $\tilde{\varepsilon} \in$ $\left[\hat{\varepsilon}_{n-1}-10^{-n}, \hat{\varepsilon}_{n-1}+10^{-n}\right.$ ) with the search ${ }_{\tilde{\varepsilon}}$ step $10^{-(n+1)}$, end (Fine estimation)

* Due to the spacing of $\beta$ between the peaks, $\gamma \neq k \beta(k=0,1, \ldots)$.

Complexity analysis: From Algorithm 1, one may realise that step 1, steps 2 and 3 , and step 4 involve $N / \gamma, 10(\gamma+1)$, and $((2 \times 10+1) q)$ times evaluation of the cost function, respectively, which in turn includes $2 L N$ number of complex multiplications (CMs). Hence, our proposed estimation method requires $2 L N(N / \gamma+10(\gamma+1)+21 q)$ number of CMs. In comparison, the computational load of the methods in [5, 6] are approximately $4 N^{3}$ and $2 L N(N+10(4(L-1)+3)+21 q)$ number of CMs, respectively. We compare our proposed method in this Letter with the ones in $[5,6]$ as they are the only available solutions in the literature applicable to GFDM without any limitations on the CFO range. Fig. 2 compares the computational complexity of our proposed method as a function of the number of subcarriers with the solutions in [5, 6] for $L=11, q=2$, and three different values of $\gamma$. We set $q=2$ to achieve estimation accuracy of $10^{-3}$ that falls well below the requirement of the LTE standard, i.e. accuracy of $2 \%$ the subcarrier spacing. Note that when $\gamma=1$, the same curve as in [7] is obtained for our proposed method. Fig. 2 shows that our proposed method leads to around an order of magnitude complexity reduction compared with the solutions in $[5,6]$ without any performance penalty as shown in the following section. From this figure, one may realise that the choice of $\gamma=L / 2$ leads to a lower complexity than when $\gamma=L$ for the values of $N$ smaller than 512. This is while for the larger values of $N$ than 512, a lower complexity can be achieved when $\gamma=L$. This is because for the small values of $N$, as $\gamma$ increases, search need to be performed for a relatively large number of local peaks in step 2. Taking this point into account, for the values of $N<512$ and $N>512$, we choose $\gamma=L / 2$ and $\gamma=L$, respectively.

Simulation results: In this section, we numerically evaluate the mean squared error (MSE) performance of our proposed method and compare it against the solutions in $[5,6]$. We consider $N=128$ and $M=2$, the subcarrier spacing of $15 \mathrm{KHz}$ and use the extended typical urban channel model (ETU) as defined in the long term evolution
(LTE) standard. Also, $\gamma=L / 2$ and the CP length is $\lfloor 0.1 N\rfloor$, i.e. long enough to accommodate the wireless channel delay spread and use a root-raised cosine prototype filter with the roll-off factor of $\alpha=0.1$, in all the simulations [6].

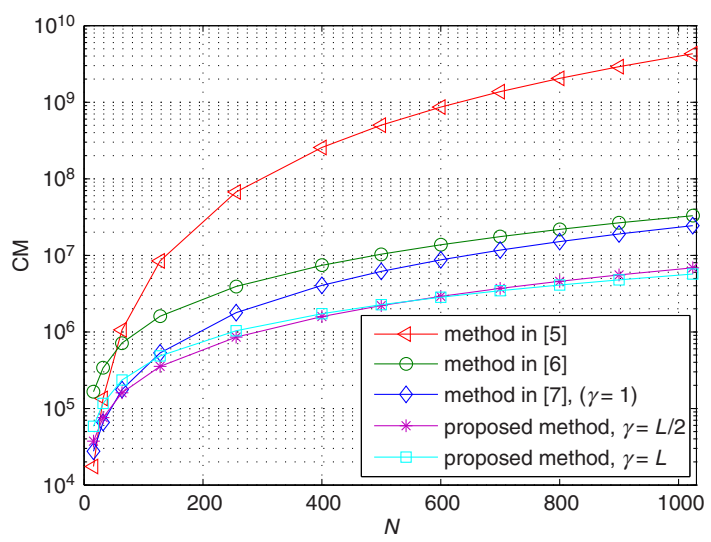

Fig. 2 Computational complexity comparison for different methods

Fig. 3 shows the MSE performance of different estimation techniques where the CFO values are chosen randomly with the uniform distribution in the range $\varepsilon \in[-(N / 2), N / 2)$. As shown in this figure, the MSE performance of our proposed estimator improves as signal-to-noise ratio (SNR) increases and is close to the relevant Cramer-Rao bound. We also compare our method with the ones in $[5,6]$ where the $\mathrm{PN}$ and $\mathrm{ZC}$ training sequences are deployed. Although our proposed method leads to the same MSE performance as the solution in [6], our proposed method has a lower computational complexity than the one in [6]. Also, the MSE of the method in [5] with PN sequence follows our proposed method for the SNRs higher than $6 \mathrm{~dB}$. This is while the method in [5] suffers from a poor performance when FD-ZC training sequence is utilised.

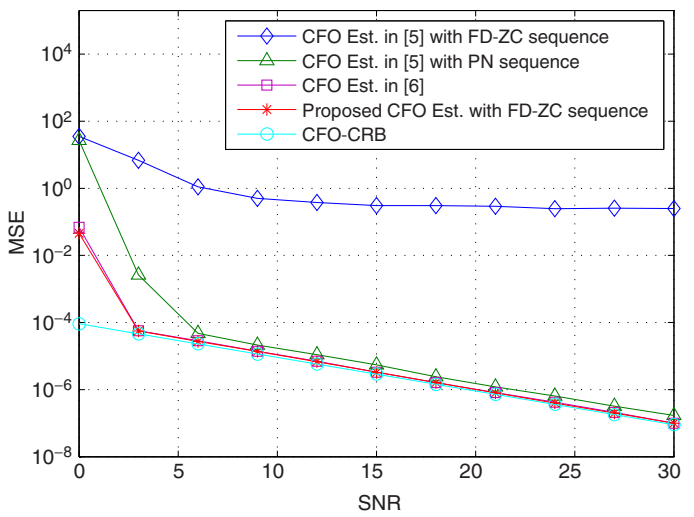

Fig. 3 MSE of the CFO estimation

Conclusion: In this Letter, we proposed a low-complexity search method for CFO estimation in GFDM. Our proposed CFO estimation method does not have any limitations on the CFO range. We showed that our proposed method brings an order of magnitude complexity reduction compared to the existing solutions in the literature with the lowest complexity without any performance penalty.

(C) The Institution of Engineering and Technology 2019

Submitted: 22 November 2018 E-first: 6 February 2019 doi: $10.1049 /$ el.2018.7800

One or more of the Figures in this Letter are available in colour online. H. Shayanfar and H. Saeedi-Sourck (EE Department, Yazd University, Yazd, Iran)

凶 E-mail: saeedi@yazd.ac.ir

A. Farhang (EE Department, Maynooth University, Maynooth, Co. Kildare, Ireland) 


\section{References}

1 Gaspar, I., Mendes, L., Michailow, N., et al.: 'A synchronization technique for generalized frequency division multiplexing'. EURASIP J. Adv. Signal Process., 2014, 2014, (67), pp. 67-76

2 Schmidl, T.M., and Cox, D.C.: 'Robust frequency and timing synchronization for OFDM', Trans. Commun., 1997, 45, pp. 1613-1621

3 Wang, P., and Lin, D.W.: 'Maximum-likelihood blind synchronization for GFDM systems', Signal Process. Lett., 2016, 23, (6), pp. 790-794

4 Na, Z., Zhang, M., Xiong, M., et al.: 'Pseudo-noise sequence based synchronization for generalized frequency division multiplexing in $5 \mathrm{G}$ communication system', Access, 2018, 6, pp. 14812-14819
5 Li, Y., Tian, B., Yi, K., et al.: 'A novel hybrid CFO estimation scheme for UFMC-based systems', Commun. Lett., 2017, 21, (6), pp. $1337-1340$

6 Shayanfar, H., Saeedi-Sourck, H., Farhang, A., et al.: 'Maximumlikelihood synchronization and channel estimation with multiuser detection in GFDMA', Trans. Emerg. Telecommun. Technol., 2018, 29, (6), pp. 1-16

7 Shayanfar, H., Saeedi-Sourck, H., and Farhang, A.: 'CFO and channel estimation techniques for GFDM'. IEEE MTT-S Int. Microwave Workshop Series on 5G (IMWS-5g), Dublin, Ireland, August 2018, pp. 1-3 